\title{
A nonlinear dynamical model of landslide evolution
}

\author{
Siqing Qin ${ }^{\mathrm{a}, *}$, Jiu Jimmy Jiao ${ }^{\mathrm{b}}$, Sijing Wang ${ }^{\mathrm{a}}$ \\ ${ }^{a}$ Engineering Geomechanics Laboratory, Institute of Geology and Geophysics, Chinese Academy of Sciences, P.O. Box 9825, \\ Beijing 100029, People's Republic of China \\ ${ }^{\mathrm{b}}$ Department of Earth Sciences, The University of Hong Kong, Pokfulam Road, Hong Kong, People's Republic of China
}

Received 1 February 2000; received in revised form 18 July 2001; accepted 20 July 2001

\begin{abstract}
A nonlinear dynamical model for the evolution of landslide is proposed. The parameters of this model are obtained through an improved iterative algorithm of inversion developed in the paper. Based on the nonlinear dynamical model and nonlinear dynamical systems (NDS) theory, the approaches to determining the Lyapunov exponents, the predictable timescale and the stability criterion of the evolutional state of landslide are given. A case study of the Xintan slope is presented to illustrate the capability and merit of the nonlinear dynamical model. C 2002 Elsevier Science B.V. All rights reserved.
\end{abstract}

Keywords: Nonlinear dynamics; Chaos; Predictable timescale; Landslide

\section{Introduction}

Specific concepts and mathematical techniques associated with nonlinear dynamical systems (NDS) theory have been widely promoted and applied in virtually every scientific discipline including seismology, geomorphology and landslide hazards (Haigh, 1988; Keilis-Borok, 1990; Phillips, 1992, 1993a, 1995; Qin et al., 1993, 2001). These concepts include chaos, fractal geometry and catastrophe theory (Phillips, 1992). The utility of NDS concepts and techniques in landslide hazards allows us to make deep insights into landslide mechanisms. NDS theory is also a way of attracting many people to pay more attention to the study of the evolutionary process of landsliding.

\footnotetext{
${ }^{*}$ Corresponding author. Fax: +86-10-620-405-74

E-mail address: qinsiqing@hotmail.com (S. Qin).
}

Deterministic chaos results in complex, irregular patterns arising from deterministic systems. Chaotic systems are sensitive to initial conditions and to perturbations. One consequence is that small errors in the specification of the initial state can be amplified rapidly (Phillips, 1993b). Considerable speculation and some evidence (Malanson et al., 1992; Phillips, 1992; Turcotte, 1992; Zeng et al., 1993) indicate that chaos may be common in geophysical phenomena. Qin et al. (1993) found that by analyzing observation series of landslides, chaos exists in the evolution process of some landslides.

Previous statistical methods of studying landslide prediction, which are of strict determinism, neglect small disturbances (tectonic, climatic, human, etc.) (Saito, 1969; Fukuzono, 1985; Voight, 1989), so chaotic behaviour in the evolution of the landslide system cannot be reflected and, hence, the long-term prediction of landslides cannot be made using these methods. In other words, the predictable timescale 
should be considered in order to forecast landslides more accurately.

NDS theory can be applied with benefit to the study of landslides. However, there are many problems to be solved in practical applications. A common approach of applying NDS theory to landslide study is as follows: write and resolve a series of dynamical equations, then study the properties of solutions obtained and finally explore the origin of all kinds of complex phenomena. However, the dynamical equations describing the evolution process of landslide have not yet been correctly written. The only information available at present is observation data and description of phenomena. That is to say, we know a series of specific solutions of the dynamical equations. If we regard such solutions as a series of discrete values of the dynamical equations, the quasiideal nonlinear dynamical equations for the evolution of landslide can, thus, be obtained through an inversion algorithm.

The aim of this paper is to provide a general framework of applying NDS theory to landslide prediction. To realize this goal, we suggest a nonlinear dynamical model for the development process of landslide, whose parameters are determined through an improved algorithm of inversion. Then, according to NDS theory, the computational method of the Lyapunov exponents, which are used to determine the predictable timescale of landslide, is given and the stability criterion reflecting the evolutional state of landslide is presented. The effectiveness of the nonlinear dynamical model is confirmed by analysis on the Xintan landslide, China.

\section{Method}

\subsection{Algorithm of inversion}

Consider the development process of a landslide as a nonlinear dynamical system (NDS). The landslide NDS includes $n$ interacting components $q_{i}$, $i=1,2, \ldots, n$. These $q_{i}$ might include, for a specific system, various factors or variables describing tectonics, lithology and hydrology, etc. The time behavior of any component is characterized by an ordinary differ- ential equation describing it as a function of the other components. Thus:

$\frac{\mathrm{d} q_{i}}{\mathrm{~d} t}=f_{i}\left(q_{1}, q_{2}, \ldots, q_{n}\right)(i=1,2, \ldots, n)$

where the function $f_{i}$ is a general nonlinear one of $q_{1}$, $q_{2}, \ldots, q_{n}$.

It is assumed that $f_{i}$ has $G_{k}$ items, and which correspond to $P_{k}$ parameters $(k=1,2, \ldots, K)$, i.e.,

$f_{i}\left(q_{1}, q_{2}, \ldots, q_{n}\right)=\sum_{k=1}^{K} G_{k} P_{k}$

If the observation data can form $M$ equations, they can be written in matrix form as:

$D=G P$

where $D=\left[q_{i}{ }^{(j+1) \Delta t}-q_{i}^{(j-1) \Delta t}\right] /(2 \Delta t)(j=2,3, \ldots$, $M+1)$ is a difference matrix with $M$ columns; $G$ is an $M \times K$ observation data matrix; and $P$ is an unknown parameter matrix with $K$ columns.

In most cases, the solutions of Eq. (2) usually are unstable when the least squares method is adopted, because $G^{T} G$ is a singular matrix or closes to a singular one and its solutions are especially sensitive to observation data errors (Huang and Yi, 1991). Bakus and Gilbert (1970) once proposed a general linear inversion algorithm to overcome this difficulty. The following is a simplified description of their algorithm of inversion.

$G^{T} G$ is a real symmetry matrix of order $K$ and its eigenvalues can be stated as:

$\left|\lambda_{1}\right| \geq\left|\lambda_{2}\right| \geq \ldots\left|\lambda_{K}\right|$

Assume that $L$ nonzero eigenvalues exist and $K-L$ eigenvalues are zero (or close to zero, its discriminant criterion is $\left|\lambda_{i}\right|<10^{-3}\left|\lambda_{1}\right|$ ), the normalized eigenvectors, corresponding to $L$ eigenvalues, can form the matrix $U$ of order $K \times L$, where $U_{i}=\left(U_{i 1}\right.$, $\left.U_{i 2}, \ldots, U_{K i}\right),(i=1,2, \ldots, L)$ are the eigenvectors corresponding to $\lambda_{i}$.

Calculating $V_{i}=\left\{1 / \lambda_{i}\right\} G U_{i}$, the matrix $V$ of order $M \times L$ can be obtained. Rearranging Eq. (2) to

$P=U B^{-1} V^{T} D$

the parameter matrix $P$ can be determined where $B$ is a diagonal matrix containing the nonzero eigenvalues. 
It has been found from practical studies (Qin et al., 1993) that when the observation period is relatively short, the predictive precision is often not satisfied using the above algorithm of inversion. For this reason, we suggest an improved iterative algorithm of inversion as follows.

As for Eq. (2), using the least squares criterion, we can obtain the following canonical equation:

$G^{T} G P=G^{T} D$

Take the parameter matrix $P$ determined from Eq. (4) as the initial estimated vector:

$P^{(0)}=\left(P_{1}^{(0)}, P_{2}^{(0)}, \ldots, P_{K}^{(0)}\right)$

and use the Gaussian-Sidel iterative formula:

$P_{i}^{(T+1)}=P_{i}^{(T)}+\frac{1}{C_{i i}}\left[e_{i}-\sum_{j=1}^{K} C_{i j} P_{j}^{(T)}\right]$

to iterate, until:

$\left|P_{i}^{(T+1)}-P_{i}^{(T)}\right|<E$

is satisfied. In Eqs. (6) and (7), $T=0,1,2 \ldots$ is the iteration number; $C_{i j}$ are the elements of the matrix $G^{T} G(i=1,2, \ldots, K) ; e_{i}$ is the column elements of the matrix $G^{T} D$; and $E$ is the permitted absolute error.

\subsection{Nonlinear dynamical analysis of the evolution of landslide}

\subsubsection{Model}

Many state variables (observation series) are probably required to describe the development process of landslides. Considering some variables are correlative, it is reasonable to select a few series with the higher confidence level as the state variables. Here, we choose three state variables to establish the nonlinear dynamical model of the evolution process of a landslide.

It is assumed that $X, Y$ and $Z$ are three different observation series, such as stress, displacement and rainfall. Considering that $X, Y$ and $Z$ are different in dimension and magnitude, they should be normalized prior to calculation. The general normalized method, for example, is that every observation value is divided by the mean value of all observation values in a series. Furthermore, we assume $f_{i}$ in Eq. (1) to be a kind of nonlinear function with regard to physical character- istics of the system. According to our recent research (Qin et al., 1993), it is assumed that $f_{i}$ has the following general forms:

$$
\begin{aligned}
\frac{\mathrm{d} X}{\mathrm{~d} t}= & a_{1} X+a_{2} Y+a_{3} Z+a_{4} X^{2}+a_{5} Y^{2}+a_{6} Z^{2} \\
& +a_{7} X Y+a_{8} X Z+a_{9} Y Z \\
\frac{\mathrm{d} Y}{\mathrm{~d} t}= & b_{1} X+b_{2} Y+b_{3} Z+b_{4} X^{2}+b_{5} Y^{2}+b_{6} Z^{2} \\
& +b_{7} X Y+b_{8} X Z+b_{9} Y Z \\
\frac{\mathrm{d} Z}{\mathrm{~d} t}= & c_{1} X+c_{2} Y+c_{3} Z+c_{4} X^{2}+c_{5} Y^{2}+c_{6} Z^{2} \\
& +c_{7} X Y+c_{8} X Z+c_{9} Y Z
\end{aligned}
$$

where $a_{1}, a_{2}, \ldots, a_{9}, b_{1}, b_{2}, \ldots, b_{9}$ and $c_{1}, c_{2}, \ldots, c_{9}$ are constants which can be solved with the above improved algorithm of inversion. The predicted values can be obtained using the Runge-Kutta integration method for Eqs. (8)-(10), and the prediction precision can be evaluated by means of the comparison between the monitored values and predicted ones.

The above nonlinear dynamical model was applied to study many landslides and earthquakes, such as Xikouzhen landslide, Wolongsi landslide, Tangshan earthquake and Haicheng earthquake, etc., in the PR China. The analytical results were satisfied.

\subsubsection{Lyapunov exponent, Kolmogorov entropy information dimension, and predictable timescale}

The Lyapunov exponents, which provide a qualitative and quantitative characterization of dynamical behavior, are related to the exponentially fast divergence or convergence of nearby orbits in phase space. A system with one or more positive Lyapunov exponents is defined to be chaotic (Wolf et al., 1985). The following is a simple introduction to solving the Lyapunov exponents from the nonlinear dynamical model (Wolf et al., 1985; Qin et al., 1993).

The Jacobi matrix $J$, composed of Eqs. (8)-(10), is:

$$
J=\left[\begin{array}{ccc}
\partial \dot{X} / \partial X & \partial \dot{X} / \partial Y & \partial \dot{X} / \partial Z \\
\partial \dot{Y} / \partial X & \partial \dot{Y} / \partial Y & \partial \dot{Y} / \partial Z \\
\partial \dot{Z} / \partial X & \partial \dot{Z} / \partial Y & \partial \dot{Z} / \partial Z
\end{array}\right]
$$

where $\dot{X}=\mathrm{d} X / \mathrm{d} t, \dot{Y}=\mathrm{d} Y / \mathrm{d} t, \dot{Z}=\mathrm{d} Z / \mathrm{d} t$. 
According to NDS theory, the Lyapunov exponent $\lambda$ can be solved from the following equation:

$\left[\begin{array}{ccc}\partial \dot{X} / \partial X-\lambda & \partial \dot{X} / \partial Y & \partial \dot{X} / \partial Z \\ \partial \dot{Y} / \partial X & \partial \dot{Y} / \partial Y-\lambda & \partial \dot{Y} / \partial Z \\ \partial \dot{Z} / \partial X & \partial \dot{Z} / \partial Y & \partial \dot{Z} / \partial Z-\lambda\end{array}\right]=0$

Further, Eq. (12) can be rewritten as:

$\lambda^{3}+A \lambda^{2}+B \lambda+C=0$

where

$$
A=-\left(\frac{\partial \dot{x}}{\partial x}+\frac{\partial \dot{y}}{\partial y}+\frac{\partial \dot{z}}{\partial z}\right)
$$

$B=\frac{\partial \dot{x}}{\partial x} \frac{\partial \dot{y}}{\partial y}+\frac{\partial \dot{x}}{\partial x} \frac{\partial \dot{z}}{\partial z}+\frac{\partial \dot{z}}{\partial z} \frac{\partial \dot{y}}{\partial y}-\frac{\partial \dot{x}}{\partial y} \frac{\partial \dot{y}}{\partial x}$

$$
-\frac{\partial \dot{x}}{\partial z} \frac{\partial \dot{z}}{\partial x}-\frac{\partial \dot{y}}{\partial z} \frac{\partial \dot{z}}{\partial y}
$$

$C=\frac{\partial \dot{x}}{\partial x} \frac{\partial \dot{y}}{\partial z} \frac{\partial \dot{z}}{\partial y}+\frac{\partial \dot{x}}{\partial y} \frac{\partial \dot{y}}{\partial x} \frac{\partial \dot{z}}{\partial z}+\frac{\partial \dot{x}}{\partial z} \frac{\partial \dot{y}}{\partial y} \frac{\partial \dot{z}}{\partial x}$

$$
-\frac{\partial \dot{x}}{\partial x} \frac{\partial \dot{y}}{\partial y} \frac{\partial \dot{z}}{\partial z}-\frac{\partial \dot{x}}{\partial y} \frac{\partial \dot{y}}{\partial z} \frac{\partial \dot{z}}{\partial x}-\frac{\partial \dot{x}}{\partial z} \frac{\partial \dot{y}}{\partial x} \frac{\partial \dot{z}}{\partial y}
$$

Three Lyapunov exponents $\left(\lambda_{1} \geq \lambda_{2} \geq \lambda_{3}\right)$ can be calculated by solving Eqs. (13)-(16). The $\lambda$ represents the exponential growth rate of state errors, which occur in a system, in the phase space along a particular direction. If the errors produced in a system gradually increase with time, it is obvious that its long-term behavior is unpredictable; if the prediction is made, it should be constrained by a certain timescale. The sum of all positive Lyapunov exponents:

$$
K_{1}=\sum_{\lambda_{j}>0} \lambda_{j}
$$

is called the Kolmogorov entropy (Liu, 1990). It represents the average production rate of information of a physical system in information theoretic terms. $1 / K_{1}$, which is called the average predictable timescale of the system, stands for the time needed for such errors to increase by one time. It is evident that this quantity is very useful for landslide prediction.

The signs of the Lyapunov exponents provide a qualitative picture of a system dynamics. The signs of three Lyapunov exponents in a three-dimensional continuous dissipative dynamical system are $(-$, $-,-),(0,-,-),(0,0,-)$ and $(+, 0,-)$ for the constant attractor, the periodical attractor, the pseudo-periodical attractor and the chaotic attractor, respectively. It can be seen that only the chaotic attractor has $\lambda_{1}>0$, which is quite different from the other attractors. Thus, according to its sign, we can decide whether the system is under the chaotic state or not.

It has been conjectured by Kaplan and Yorke (Wolf et al., 1985) that the Lyapunov information dimension $d$ is related to the Lyapunov spectrum by the equation:

$d=T+\sum_{i=1}^{T} \frac{\lambda_{i}}{\left|\lambda_{T+1}\right|}$

where $T$ is equal to the largest positive integer which satisfies $\lambda_{1}+\lambda_{2}+\ldots \lambda_{T} \geq 0$. The information dimensions of the constant, periodical and pseudo-periodical attractors are obviously integers, 0,1 and 2, respectively, whereas the information dimension of the chaotic attractor is decimal. That is another indication on which we can rely to judge the state of the system.

\subsubsection{Criterion for the stability of the system}

The Routh-Hurwitz criterion (Puccia and Levins, 1985) states that the necessary and sufficient conditions for the stability of the system are:

$A>0, A B>C, C>0$

where $A, B$ and $C$ are defined in Eqs. (14)-(16). If Eq. (19) is satisfied, the evolutional state of landslide is stable; otherwise, it is unstable.

\section{Case study}

The Xintan landslide, which occurred on 12 June 1985, is located at Xintan town (Fig. 1) in the 


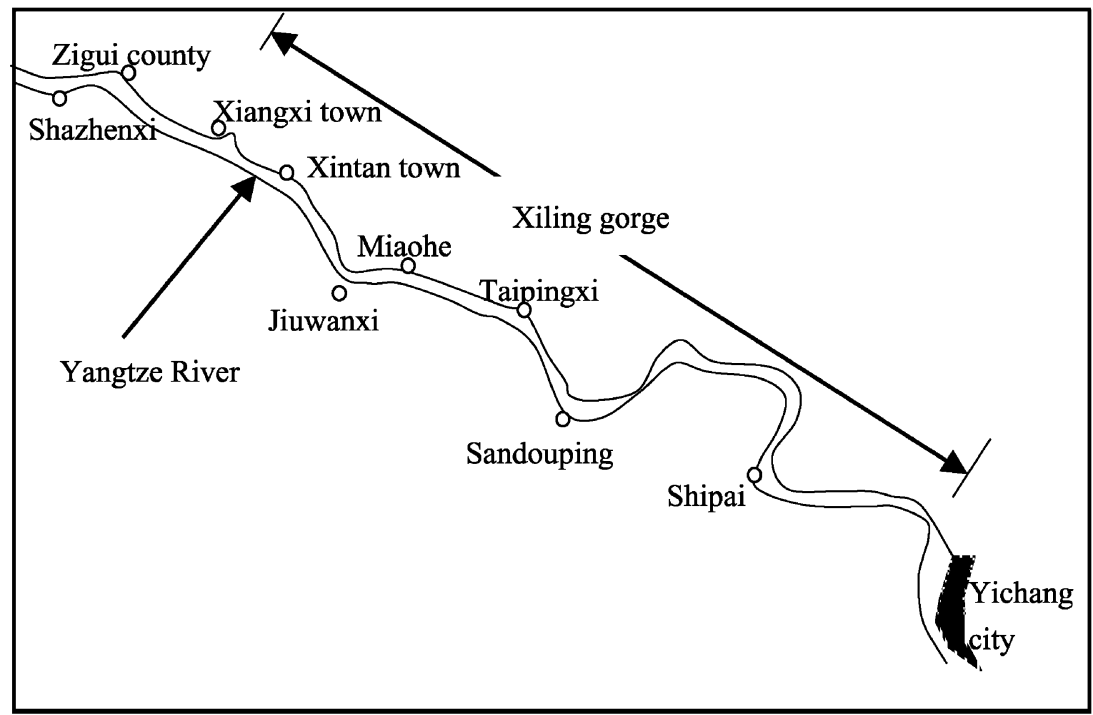

Fig. 1. Site map of Xintan landslide.

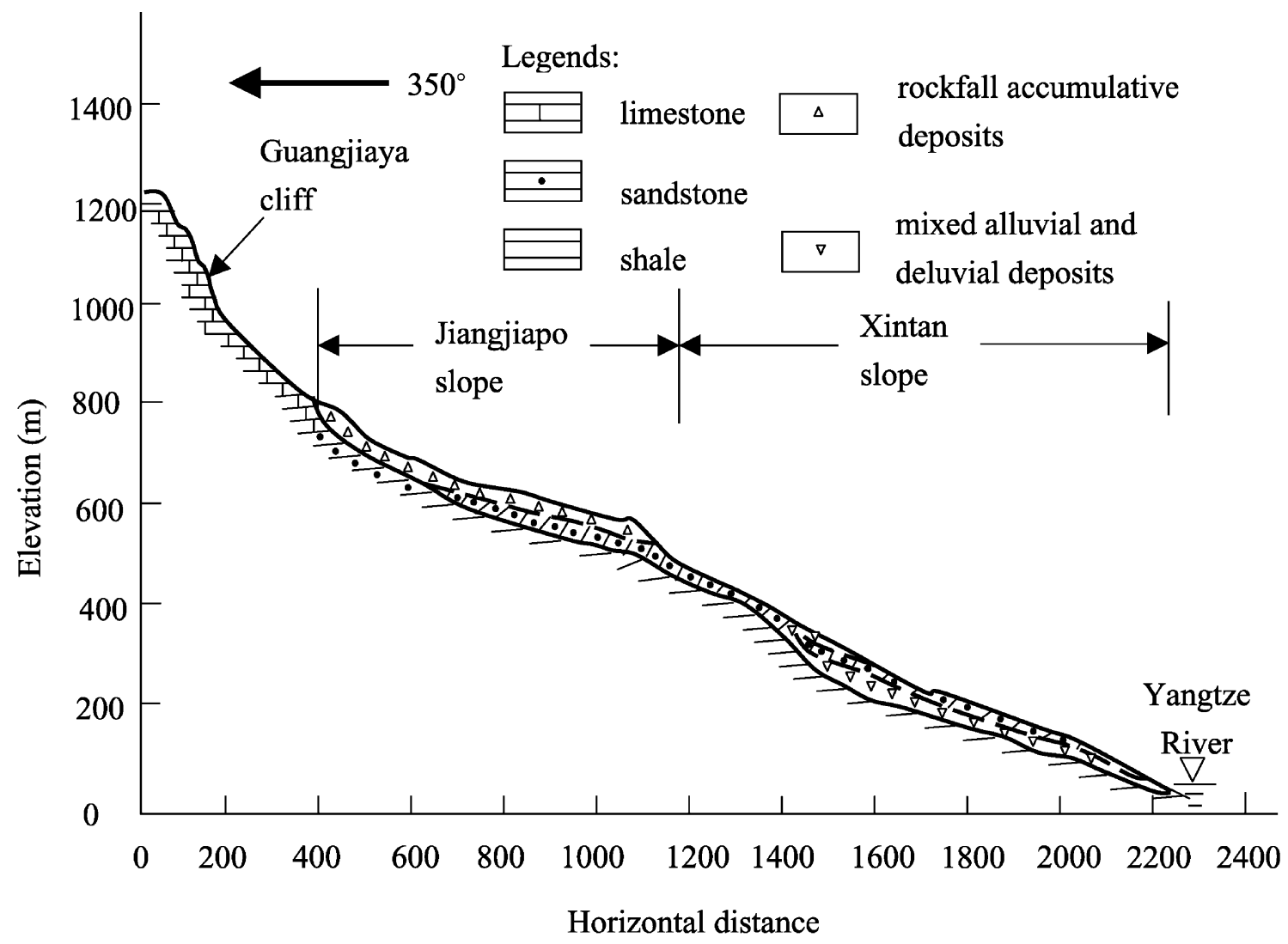

Fig. 2. Geological section of Xintan landslide. 
Xiling gorge, Yangtse River, PR China. It is a landslide of accumulative deposits of $20 \times 10^{6} \mathrm{~m}^{3}$. The landslide mass covers the Middle Silurian sandstone and Late Silurian shale. The slope of landslide may be divided in to two sections (Fig. 2). The upper section is Jianjiapo slope and the lower part is Xintan slope. The Jiangjiapo slope is composed of rockfall accumulative deposits, containing limestone fragments and debris of violet sandstone and shale. The section of Xintan slope is composed of mixed alluvial and colluvial deposits containing mainly fragments and debris of limestone, imbedded with gravel and lenses of clay. Groundwater was found at the front of the Xintan slope in the form of springs.

Field studies were carried out to understand the morphology and geology. The rockfall from the Guangjiaya cliff loaded over the unstable slope of Jiangjiapo for a long period. Also, rainfall played an important role. The geological investigation showed that the Jianjiapo slope pushed the Xintan slope to slide. At the beginning of the rain season in May, 1985, the displacements were found to be much accelerated, reaching to $200-300 \mathrm{~mm} /$ day. Finally in the morning of 12 June 1985, a major high-speed slide happened.

In April 1988, it was found that the Xintan slope body had begun to redeform The deformation, underground water level and geo-stress were observed using an automatic monitoring system. Owing to the absence of complete data records at the other observation points, we analyze only the data at $5^{\#}$ observation point. The monitored values of crack width $(X)$, underground water level $(Y)$ and horizontal geo-stress $(Z)$ at $5^{\#}$ observation point from May 1988 to February 1990 are shown in Fig. 3 (Qin et al., 1993).

We normalize the monitored values and then determine unknown parameters in Eqs. (8)-(10) using the above-mentioned improved algorithm of inversion. The allowable absolute error of iteration is

Fig. 3. Comparison between monitoring values and forecasting ones. (a) Crack width $(X)$ versus time $(t)$, (b) underground water level $(Y)$ versus time $(t)$ and (c) geo-stress $(Z)$ versus time $(t)$.
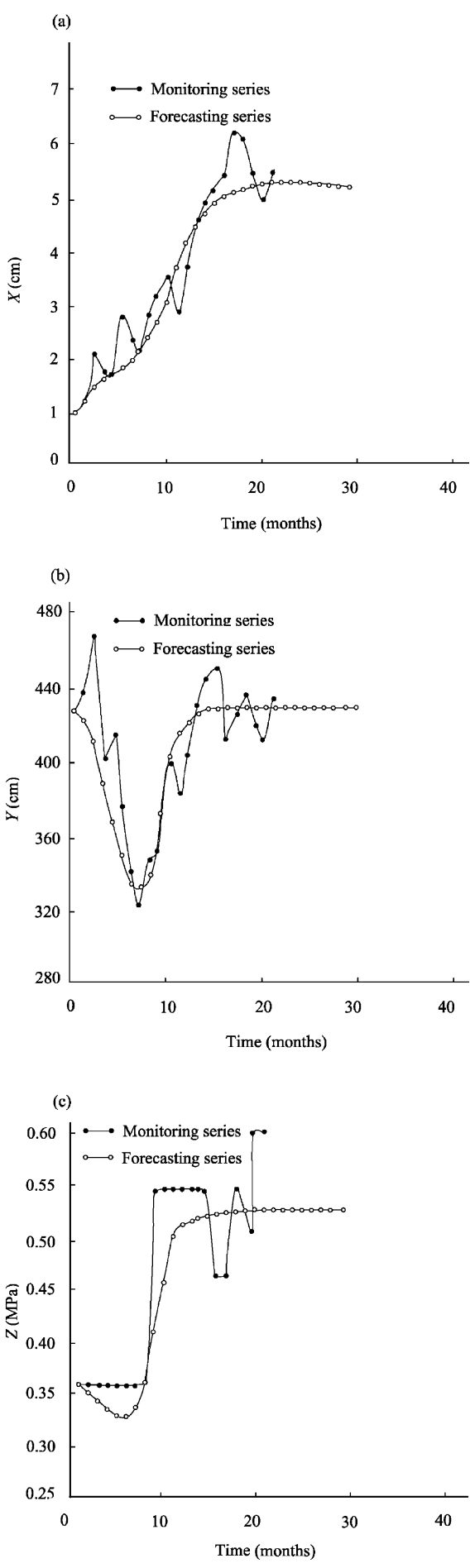
$E=10^{-4}$, the number of iterations is 35000 . The nonlinear dynamical equations obtained are as follows:

$$
\begin{aligned}
\frac{\mathrm{d} X}{\mathrm{~d} t}= & -1.0320 X-3.5487 Y+4.1215 Z \\
& +0.1360 X^{2}+0.0784 Y^{2}-3.0090 Z^{2} \\
& +1.3044 X Y-0.9707 X Z+3.0576 Y Z \\
\frac{\mathrm{d} Y}{\mathrm{~d} t}= & 0.8787 X-2.1705 Y+1.6519 Z+0.1047 X^{2} \\
& +0.5600 Y^{2}-1.6399 Z^{2}-1.1660 X Y \\
& +0.0319 X Z+1.7832 Y Z \\
\frac{\mathrm{d} Z}{\mathrm{~d} t}= & 1.5146 X-2.4316 Y+1.6311 Z+0.1547 X^{2} \\
& +0.9064 Y^{2}-1.2434 Z^{2}-1.0064 X Y \\
& -0.7451 X Z+1.2895 Y Z
\end{aligned}
$$

The predicted values (Fig. 3) can be obtained using the Runge-Kutta integration method for Eqs. (20)(22). The average relative errors between the predicted values and the original ones for $X, Y$ and $Z$ series are $7 \%, 6 \%$ and $10 \%$, respectively. This shows that the nonlinear dynamical model of the Xintan landslide established fits well with the observed data.

Changes of the Lyapunov exponents $\lambda_{i}(i=1,2,3)$ versus time are shown in Fig. 4. It is obvious that the symbol of $\lambda_{i}$ has such a time-dependent change: $(+,+$, $-) \rightarrow(+, 0,-) \rightarrow(+,-,-) \rightarrow(0,-,-) \rightarrow(-$, $-,-)$. This change means that the slope body is evolved from a chaotic motion through a periodical one, finally to a deterministic one - a process full of uncertainty and complexity.

$K_{1}$ can be calculated from formula (17). The mean value of $K_{1}$ is 0.73 . Thus, the average predictable timescale is 1.37 months. This means that the deterministic prediction can be carried out in the period from March 1990 to 11 April 1990. Beyond this period, the prediction error will become large.

Fig. 4. Variation of the Lyapunov exponents versus time. (a) Maximum Lyapunov exponent $\left(\lambda_{1}\right)$ versus time $(t)$, (b) medium Lyapunov exponent $\left(\lambda_{2}\right)$ versus time $(t)$ and (c) minimum Lyapunov exponent $\left(\lambda_{3}\right)$ versus time $(t)$.
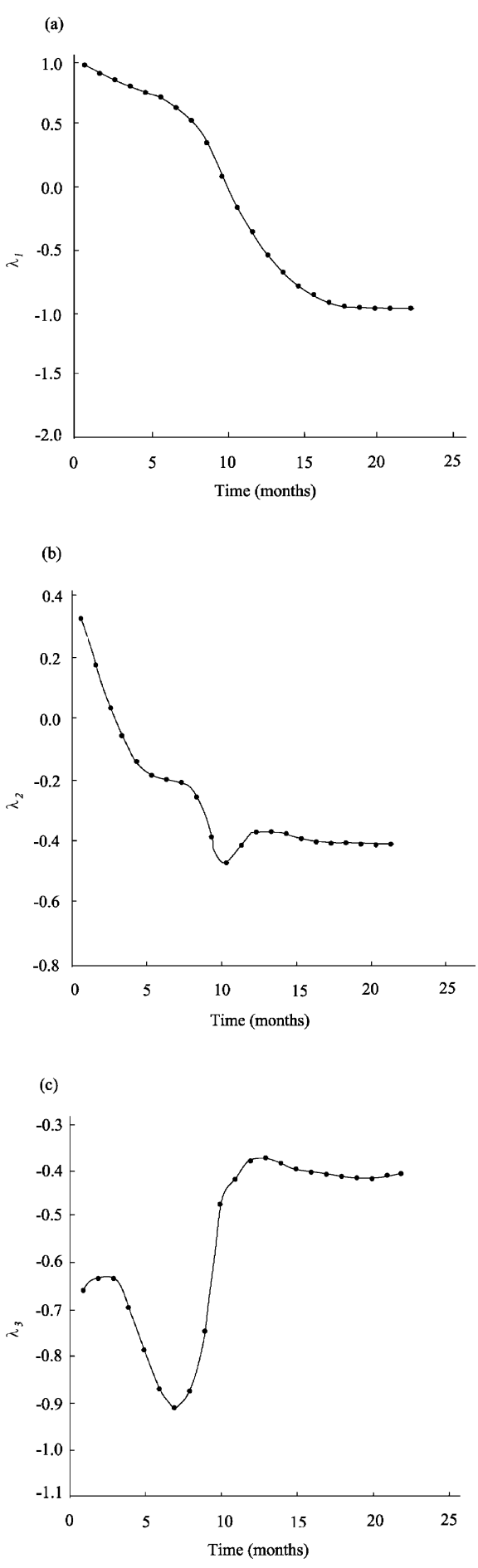


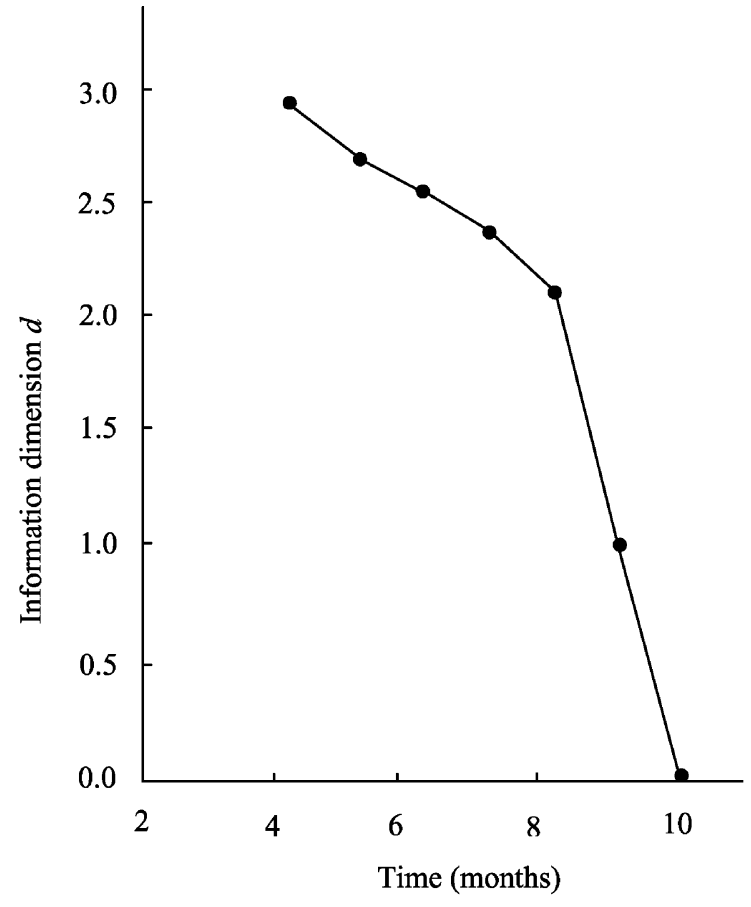

Fig. 5. Variation of the Lyapunov information dimension $d$ versus time $t$.

The variation of the Lyapunov information dimension $d$ versus time is illustrated in Fig. 5. We find that there is a dimension-decreased and ordered phenomenon, similar to rock deformation, in the evolution process of the slope. The results of stability analysis on the Xintan slope body show that it evolves towards a stable state, which is consistent with the fact that the slope is currently stable.

\section{Conclusions}

A nonlinear dynamical model for the evolution of landslides is suggested. The parameters of this model can be determined by using an improved iteration algorithm of inversion developed in this paper. The methods of calculating the Lyapunov exponents, the Kolmogorov entropy, the Lyapunov information dimension, predictable timescale and stability criterion from the nonlinear dynamical model are also given.

When chaos exists in the evolution of landslide, the predictable timescale must be considered for forecasting landslide behavior. The NDS analysis on the
Xintan landslide shows that its evolutionary process is very complicated, i.e., sometimes periodic, sometimes chaotic, and sometimes deterministic. This implies that any statistical prediction model for landslide must involve these properties to make objective appraise on the evolution of landslide.

This paper presents a NDS theoretical framework that explicitly links observational data and a nonlinear model of landslide formation with the mathematical properties of chaotic systems. Further work, such as determination of the specific expression $f_{i}$, should be done, in order to get better prediction results.

\section{Acknowledgements}

The study is supported by the Croucher foundation of Hong Kong, the programme for the talents by Chinese Academy of Sciences (CAS) and the environment and resource program (KZ952-J1-416), CAS.

\section{References}

Bakus, G., Gilbert, F., 1970. Uniqueness in the inversion of inaccurate gross earth data. Philos. Trans. R. Soc. London, Ser. A 266 (1173), 123-192.

Fukuzono, T., 1985. A new method for predicting the failure time of a slope. Proc. IV International Conference and Field Workshop on Landslide, Tokyo, 1985. Tokyo University Press, Tokyo, pp. 12-20.

Haigh, M.J., 1988. Dynamic systems approaches in landslide hazard research. Z. Geomorphol. N. F. Suppl.-Bd. 67, 79-91.

Huang, J.P., Yi, Y.H., 1991. Nonlinear dynamics model inversed with observation data. Sci. China, Ser. B 3, 331-336.

Keilis-Borok, V.I., 1990. The lithosphere of the earth as a nonlinear system with implications for earthquake prediction. Rev. Geophys. $28,19-34$.

Liu, S.D., 1990. Simulation on the earth system and chaotic timeseries (in Chinese). J. Geophys. 33, 144-152.

Malanson, G.P., Butler, D.R., Georgakakos, K.P., 1992. Non-equilibrium geomorphic processes and deterministic chaos. Geomorphology 5, 311-322.

Phillips, J.D., 1992. Nonlinear dynamical systems in geomorphology: revolution or evolution? Geomorphology 5, 219-229.

Phillips, J.D., 1993a. Instability and chaos in hillslope evolution. Am. J. Sci. 293, 25-48.

Phillips, J.D., 1993b. Spatial-domain chaos in landscapes. Geogr. Anal. 25, 101-117.

Phillips, J., 1995. Nonlinear dynamics and the evolution of relief. Geomorphology 14, 57-64. 
Puccia, C.J., Levins, R., 1985. Qualitative Modeling of Complex Systems. Harvard Univ. Press, Cambridge, MA, pp. 61-70.

Qin, S.Q., Zhang, Z.Y., Wang, S.Q., Huang, R.Q., 1993. An Introduction to Nonlinear Engineering Geology. Southwest University Press of Transportation, Chengdu, PR China, pp. 50-90 (in Chinese).

Qin, S.Q., Jiao, J.J., Wang, S.J., 2001. The predictable timescale of landslides. Bull. Eng. Geol. Environ. 59, 307-312.

Saito, M., 1969. Forecasting time of slope failure by tertiary creep. Proc. Seventh Int. Conf. On Soil Mech. and Found. Engineering, Montreal, 1969, Pergamon Press, Oxford, pp. 667-683.
Turcotte, D.L., 1992. Fractals and Chaos in Geology and Geophysics. Cambridge Univ. Press, New York, p. 221.

Voight, B., 1989. Materials science law applies to time forecasts of slope failure. Landslide News 3, 8-11.

Wolf, A., Swift, J.B., Swinney, H.L., Vastano, J.A., 1985. Determining Lyapunov exponents from a time series. Physica 16D, $285-317$.

Zeng, X., Pielke, R.A., Eykholt, R., 1993. Chaos theory and its applications to the atmosphere. Bull. Am. Meteorol. Soc. 74, 631-644. 